\title{
Temporally Dynamic Interaction between Drug Cue Reactivity and Response Inhibition: An fMRI Study among People with Methamphetamine Use Disorder
}

\section{Sara Jafakesh}

Shiraz University of Technology

Arshiya Sangchooli

Iranian National Center for Addiction Studies (INCAS), Tehran University of Medical Science

Ardalan Aarabi

University of Picardie Jules Verne

Mohammad Sadegh Helfroush

Shiraz University of Technology

Amirhossein Dakhili

Tehran University of Medical Sciences

Mohammad Ali Oghabian

Iran University of Medical Sciences

Kamran Kazemi ( $\nabla$ kazemi@sutech.ac.ir)

Shiraz University of Technology

Hamed Ekhtiari

Laureate Institute for Brain Research (LIBR)

\section{Research Article}

Keywords: Methamphetamine, fMRI, Cue-reactivity, Craving, Response-inhibition, Go-NoGo, Addiction, interaction, Temporal dynamics

Posted Date: August 26th, 2021

DOl: https://doi.org/10.21203/rs.3.rs-712109/v1

License: (c) (i) This work is licensed under a Creative Commons Attribution 4.0 International License.

Read Full License 


\section{Abstract}

Cue-induced drug craving and disinhibition are two essential components of continued drug use and relapse in substance use disorders. While these two phenomena develop and interact across time, the temporal dynamics of their underlying neural activity and their interaction remain under-investigated. To explore these dynamics, an analysis of time-varying activation was applied to fMRI data from 62 men with methamphetamine use disorder in their first weeks of recovery in abstinence-based treatment program. Using a mixed block-event, factorial cue-reactivity/Go-NoGo task, and a sliding window across the task duration, dynamically-activated regions were identified in linear mixed effects models (LMEs). Habituation to drug cues across time was observed in the superior temporal gyri, amygdalae, left hippocampus, and right precuneus, while response-inhibition was associated with the sensitization of temporally-dynamic activations across many regions of the inhibitory frontoparietal network. Cuereactivity and response-inhibition dynamically interact in the parahippocampal gyri and right precuneus (corrected p-value $<0.001$ ) regions, which show a declining cue-reactivity contrast and an increasing response-inhibition contrast. Overall, the declining craving-related activations (habituation) and increasing inhibition-associated activations (sensitization) along the task duration suggest the gradual recruitment of response-inhibition process and the concurrent habituation to drug cues in areas with significant dynamic interaction. This exploratory study demonstrates the time-variance of the neural activations undergirding cue-reactivity, response-inhibition, and their interaction, and suggests potentials to assess this dynamic interaction. This preliminary evidence provides justifications for new avenues in biomarker development and interventions using cue exposure paradigms, which could promote habituation to drug cues and sensitization in inhibitory control regions.

\section{Introduction}

The prevalence and health burden of methamphetamine use disorder (MUD) continues to increase globally ${ }^{1}$ and in countries such as the US, where $0.4 \%$ of the adult population suffers from methamphetamine use disorder ${ }^{2}$ and methamphetamine-related overdose rates have tripled from 2011 to $2016^{3}$. This potential crisis is compounded by the fact that despite decades of research, assessments of MUD are still largely reliant on interviews, self-reported measures, and urinalysis 4,5 , and data on effective interventions for MUD remains inconsistent ${ }^{6}$, with growing calls to better delineate the neurobiology of MUD to identify novel treatment targets and clinically-relevant biomarkers ${ }^{7}$. In tandem with research elucidating the involvement of a plethora of cognitive functions in the MUD ${ }^{8}$, functional magnetic resonance imaging (fMRI) studies on the neurobiology of MUD have characterized a number of functional brain changes associated with cognitive alterations in the MUD, methamphetamine craving, use history and relapse risk, and treatment outcomes 9,10 .

Two central aspects of methamphetamine use disorder are a characteristic reactivity to drug cues (itself involving attentional bias towards drug cues, their increased salience, and ultimately the induction of craving) ${ }^{11,12}$, and failures of executive control and response-inhibition ${ }^{13}$. These phenomena widely 
figure in models of a substance use disorder, such as the "impaired response-inhibition and salience attribution" model ${ }^{14}$ and dual-process models, which comprise automatic approach behavior towards substances and reduced abilities to inhibit these behaviors ${ }^{15}$. An increasing number of task-based fMRI studies in MUD have investigated the neural correlates of either methamphetamine cue-reactivity, using conventional cue exposure tasks ${ }^{16-19}$ or cognitive control and response-inhibition, using varieties of Stroop, Stop Signal or Go-NoGo tasks ${ }^{20-22}$, but a potentially more promising approach has been to assess response-inhibition concurrently with cue-reactivity. These latter studies are motivated by the interrelationship between response-inhibition and cue-reactivity, with drug cue exposure hampering inhibitory control ${ }^{23-25}$ and poor inhibitory control precipitating higher induced craving ${ }^{26}$. The fMRI research in this area typically involves mixed tasks to investigate the interaction of drug cue processing and response-inhibition, such as Go-NoGo tasks in which Go and NoGo signals are independently mixed through neutral and substance cues ${ }^{27,28}$ or in which substance cues are themselves the NoGo signal 29,30 .

A notable characteristic of the above-mentioned studies is an assumption of time-invariant voxel-wise or regional activation, whereby in both response-inhibition and cue-reactivity tasks average responses are obtained across the entire task duration. This conventional "static" approach might be problematic in light of the evidence that activation patterns during exposure to emotionally salient stimuli are often dynamic and vary across the task duration, for example when brain regions demonstrate various patterns of habituation to emotionally negative cues or reward stimuli ${ }^{31-34}$ which might be explained through extinction learning ${ }^{35}$, though regional sensitization to pictures of angry faces has also been observed ${ }^{36}$. Considering the potentially multiphasic nature of the cue-reactivity process which unfolds over seconds and minutes and primarily involves different regions and networks at each stage ${ }^{11}$, it's not surprising that three recent studies on the MUD and opioid use disorder have found evidence of temporally dynamic activation patterns during cue-reactivity in regions such as the amygdala, the dorsal anterior cingulate cortex, the ventromedial prefrontal cortex, the ventral striatum, the caudate nuclei and insular cortices, and various prefrontal regions ${ }^{37-39}$. Given the temporally dynamic involvement of regions such as the bilateral motor and prefrontal cortices in response-inhibition ${ }^{40}$ and the dynamic reconfiguration of functional brain networks ${ }^{41}$, it's reasonable to expect similarly dynamic activation patterns to be implicated in successful and dysfunctional response-inhibition in individuals with substance use disorders.

Using data obtained from the first fMRI implementation of a novel mixed cue-reactivity/Go-NoGo task, this study aims to investigate the dynamic brain activation patterns that underlies methamphetamine cue-reactivity, response-inhibition, and their interaction in individuals with MUD. After the identification of regions with dynamic (i.e., time-variant) activation and the characterization of activation patterns in these regions, the correlation of sensitization/habituation slopes in these regions with behavioral variables is estimated to further explore the validity of this dynamic regional activity. 


\section{Materials And Methods \\ Participants}

Sixty-two men with MUD (age: $32.12 \pm 5.89$ ) were recruited from addiction treatment centers in Tehran, Iran. Inclusion criteria were (1) Diagnosis of methamphetamine dependence (for at least 6 months) according to the Diagnostic and Statistical Manual of Mental Disorders, Fourth Edition criteria (DSM-IV TR) ${ }^{42}$, (2) abstinence from any substance for at least one week, with the exception of nicotine, based on self-report and confirmed by urine drug screening, (3) right-handedness, determined using the Edinburgh Handedness Inventory ${ }^{43}$, and (4) age between 20 and 40 years. Exclusion Criteria were (a) any comorbid axis-I disorders other than drug dependence, based on DSM-IV TR criteria, (b) ineligibility for MRI scanning (e.g., metal implants, claustrophobia), (c) history of head trauma resulting in neurological disorders. Nine participants were excluded from fMRI analyses due to excessive movement during scanning, leaving 53 individuals (see Preprocessing section for details). Demographic and behavioral data of the 53 participants who were included in the analyses are provided in Table 1.

The research protocol was designed and implemented in accordance with the Declaration of Helsinki. After a referral from treatment centers to the research team, individuals were informed regarding the aims of the project, the collected information and measures are taken to ensure anonymity, scanning procedures, the $\mathrm{fMRI}$ task and its potential to induce methamphetamine craving, and that they can exit the study at any point with no implications for their ongoing treatment. After the consent form was read both out loud by a psychologist and by the individual to ensure comprehension, participants provided written, informed consent prior to further screening for enrollment. The data collected from each participant was sent to the primary data analyst and anonymized before further processing. The study protocol was reviewed and approved by the ethical review board of the Tehran University of Medical Sciences with the approval code 93-02-98-23869.

\section{Procedures and measures}

Participants were abstinent prior to scanning, but were allowed to smoke. After arriving at the imaging center, participants were interviewed by two clinical psychologists, and several measures were administered prior to scanning. Collected data included demographic information, mental status examination, clinical assessments (including drug use profile, treatment history), risky behaviors profile, the Barratt Impulsiveness Scale-11 (BIS-11) ${ }^{44}$, the Depression Anxiety and Stress Scale-21 (DASS-21) 45 , and the Positive and Negative Affect Schedule (PANAS) ${ }^{46}$. Methamphetamine craving was assessed using a 0-100 Visual Analog Scale (VAS) before MR scanning. After scanning, participants were again assessed with PANAS and rated their craving (Table 1). To minimize the risk of drug use after the fMRI session, participants were asked to remain in the scanning center for an hour while recovering.

\section{fMRI Go-NoGo task}


Participants were scanned during four consecutive runs of the mixed Go-NoGo task, separated by resting blocks with a fixation point. Each run included four 36-second blocks of 24 stimuli, depicting geometric Go-NoGo signs overlaid on background cues. Background images were either blank (black), neutral images, negative emotional cues, or methamphetamine-related cues. Each block contained 18 Go signs (triangles, squares, or diamonds) and 6 NoGo signs (circles). Each stimulus lasted one second and was followed by a jittered inter-stimulus interval generated using a gamma probability density function (mean $=0.5$ ). The blocks were separated by 18-second fixation periods in which a white cross was shown on a black background, so each run took 198 seconds. A total of 16 blocks were presented, four of each condition (blank, neutral, negative, drug). The total scanning duration was approximately 13 minutes (Fig. 1).

Participants were instructed to respond as fast as possible when the Go stimuli were presented and to withhold their response to NoGo stimuli. Participants underwent a training test outside the scanner and were informed that both speed and accuracy are important.

The methamphetamine cues have been evaluated in previous studies of Iranian participants 47 , and neutral and negative emotional cues were selected from the IAPS database ${ }^{48}$. The researchers had permission to use the utilized images. Neutral, methamphetamine, and negative cues were matched in terms of visual complexity, brightness, luminance, and color.

Table 1

Demographic and the profile of Methamphetamine users $(n=53)$

Values are reported with mean (standard deviation) or frequency (\%) format. 


\begin{tabular}{|ll|}
\hline Variable & Mean (SD) \\
\hline Age (years) & $32.05(5.2)$ \\
\hline Education (years) & $10.23(2.86)$ \\
\hline Age of Meth use onset & $24.3(5.77)$ \\
\hline Meth use duration (years) & $7.46(7.3)$ \\
\hline Dosage of Meth (gram per day) & $11.64(14.46)$ \\
\hline Cost of Meth (Dollar per month) & $467.3(390.94)$ \\
\hline Clinical Scales & Mean (SD) \\
\hline Pre-scanning Visual Analog Scale (VAS) (0-100) & $32.87(35.16)$ \\
\hline Post-scanning Visual Analog Scale (VAS) (0-100) & $30.7(37.03)$ \\
\hline Depression Anxiety and Stress Scale (DASS) (0-60) & $27.47(13.63)$ \\
\hline Barratt Impulsiveness Scale, motor score (BIS-Motor) (0-100) & $26.98(6.34)$ \\
\hline Barratt Impulsiveness Scale, total score (BIS-Sum) (0-100) & $75.32(13.62)$ \\
\hline Days of Drug use in the last month (before current treatment) & Frequency (\%) \\
\hline Methamphetamine & $100 \%$ \\
\hline Opioid & $77.7 \%$ \\
\hline Cannabis & $52.7 \%$ \\
\hline Alcohol & $32.26 \%$ \\
\hline Sedatives & $21.2 \%$ \\
\hline Hallucinogens & $8.88 \%$ \\
\hline Cocaine & $8.36 \%$ \\
\hline
\end{tabular}

\section{fMRI Go-NoGo task}

Participants were scanned during four consecutive runs of the mixed Go-NoGo task, separated by resting blocks with a fixation point. Each run included four 36-second blocks of 24 stimuli, depicting geometric Go-NoGo signs overlaid on background cues. Background images were either blank (black), neutral images, negative emotional cues, or methamphetamine-related cues. Each block contained 18 Go signs (triangles, squares, or diamonds) and 6 NoGo signs (circles). Each stimulus lasted one second and was followed by a jittered inter-stimulus interval generated using a gamma probability density function (mean $=0.5$ ). The blocks were separated by 18 -second fixation periods in which a white cross was shown on a 
black background, so each run took 198 seconds. A total of 16 blocks were presented, four of each condition (blank, neutral, negative, drug). The total scanning duration was approximately 13 minutes (Fig. 1).

Participants were instructed to respond as fast as possible when the Go stimuli were presented and to withhold their response to NoGo stimuli. Participants underwent a training test outside the scanner and were informed that both speed and accuracy are important.

The methamphetamine cues have been evaluated in previous studies of Iranian participants 47 , and neutral and negative emotional cues were selected from the IAPS database ${ }^{48}$. The researchers had permission to use the utilized images. Neutral, methamphetamine, and negative cues were matched in terms of visual complexity, brightness, luminance, and color.

\section{Scanning parameters}

Whole-brain T2* weighted images were acquired in a 3.0 Tesla Siemens (MAGNETOM Trio; Germany) scanner in the Tehran University of Medical Sciences, Iran. Functional scans were collected using a 2D gradient echo EPI sequence, and each volume was comprised of 40 contiguous axial slices $(T R=2.2 \mathrm{~s}$, $\mathrm{TE}=30 \mathrm{~ms}$, field of view $(\mathrm{FOV})=192 \times 192$, in-plane voxel size $3.0 \mathrm{~mm} \times 3.0 \mathrm{~mm}$, slice thickness $3 \mathrm{~mm}$, $\mathrm{FA}=90^{\circ}$ ). The scanning session lasted 806 seconds. A high-resolution T1-weighted structural image was also acquired for each participant for co-registration during preprocessing and to exclude participants with any structural abnormality. Structural images were acquired through a sagittal T1-weighted magnetization-prepared rapid acquisition (MP-RAGE) sequence with the following parameters: repetition time $=1800 \mathrm{~ms}$, echo time $=3.44 \mathrm{~ms}, \mathrm{FOV}=256 \mathrm{~cm} \times 256 \mathrm{~cm}$, flip angle $=7^{\circ}, 1 \mathrm{~mm} 3$ Voxels.

\section{Pre-processing}

FSL (FMRIB's Software Library, www.fmrib.ox.ac.uk/fsl) version 6.0.3 was used to preprocess structural and functional data ${ }^{49}$. Structural data was skull-stripped to remove non-brain tissue from the structural T1-weighted images using the Brain Extraction Tool (BET). BET parameters were chosen based on each individual skull size.

Functional data were analyzed using the fMRI Expert Analysis Tool (FEAT), part of FMRIB's Software Library. The functional pre-processing included the removal of the first five volumes, motion correction with 6 degrees of freedom, interleaved slice-timing correction, linear Boundary-Based Registration (BBR) of functional images to the high-resolution T1 images, nonlinear registration of the T1 images to the standard Montreal Neurological Institute (MNI) space with 12 degrees of freedom, intensity normalization, smoothing with a 5-mm full-width at half-maximum (FWHM) Gaussian kernel, denoising with melodic ICA, high-pass temporal filtering (with the cut-off frequency equal to the inverse of 120 seconds). 
High motion effects on fMRI time series were identified using the DVARS metric ${ }^{50}$ and were regressed out in the first-level generalized linear model (GLM) analysis. "High movement subjects" were defined as those with displacement $>4 \mathrm{~mm}$ and also DVARS > 75 in more than ten volumes in a single block (36sec), and were excluded from the analyses ( 9 subjects).

\section{Conventional whole-brain analysis}

The pre-processed functional images were analyzed in a GLM framework. Event-types were specified at the time of indicator onset, and the canonical hemodynamic response was used to model the regressors for the conditions of interest. The event types included Neutral Successful NoGo (NSNG), Neutral Successful Go (NSG), Drug Successful NoGo (DSNG) and Drug Successful Go (DSG), blank and negativeemotional successful Go and NoGo trials, and unsuccessful Go and NoGo trials, were included in the GLM as independent regressors. Six head motion parameters and high motion time-points extracted based on the DVARS metric were included as nuisance regressors.

To determine the time-invariant neural correlates of methamphetamine cue-reactivity, response-inhibition and response-inhibition during cue exposure, each event type was included as a single regressor and three contrasts were defined: $(D S N G+D S G)>(N S N G+N S G)$ to model cue-reactivity, $(D S N G+N S N G)>$ $(D S G+N S G)$ to model inhibition and $(D S N G>D S G)>(N S N G>N S G)$ to model the interaction of cuereactivity and inhibition.

To calculate average activations patterns, first-level models were then carried forward into a second-level mixed effects analysis using FMRIB's Local Analysis of Mixed Effects (FLAME) tool with a cluster defining threshold (Z-threshold >3.1, corrected cluster-level threshold: $p<0.001$ ).

\section{Temporally dynamic fMRI analysis}

ROI-based whole-brain analyses were performed using the Brainnetome atlas (BNA) ${ }^{51}$. First, the whole brain was parcellated into 246 regions based on the BNA. The BNA masks in MNI space were then registered to each subject's space using the transformation matrices derived from the pre-processing step and after determining subject-specific masks for each ROI across the 53 subjects, the mean activations and standard errors were calculated. We then used a sliding window over the BOLD response to investigate temporal variability, with a window duration of two task runs (396 seconds) and a sliding interval equal to one run (198 seconds) leading to the extraction of three overlapping windows. We modeled each of the four event types with separate regressors across the three windows, so every participant had six beta coefficients estimated for each of the three contrasts (cue-reactivity, inhibition, and interaction).

Then, three Linear Mixed Effects (LME) models were fit to the fMRI data in R, version 3.6.2 ${ }^{52}$. The models were all specified as "beta condition * time" with the condition, time, and their interaction as fixed effects and the subject as a random effect. The conditions were $(D S N G+D S G)$ or $(N S N G+N S G)$ in the 
cue-reactivity LME, $(D S N G+N S N G)$ or $(D S G+N S G)$ in the inhibition LME and (DSNG > DSG) or (NSNG > $N S G)$ for the interaction LME. Time was treated as a discrete variable with integer values of 1 through 3 (for the first through the third window) which were mean centered. For each of the three models, regions with a significant main effect of time and condition and those with a significant condition-by-time interaction were identified after a False Discovery Rate (FDR) correction with a threshold of $p<0.001$. Lastly, for each model, the temporal activation dynamics of significant regions were examined by plotting the beta values for the relevant conditions across the three windows.

\section{Correlation of dynamic activity and behavioural data}

For every ROI exhibiting a significant condition-by-time interaction in each model, separate "beta condition * time" linear models (LMs) were fit for each subject and the slope of the interaction term was taken to be an index of the evolution of condition-relative individual-level dynamic activity in the ROI. For cue-reactivity LMs the slope would represent sensitization to drug versus neutral cues across time, in inhibition LMs it would represent temporally escalating activation when inhibiting versus not inhibiting pre-potent responses, and in interaction LMs it would be a subject-level reflection of an increased neural load required to successfully inhibit responses in the presence of drug versus neutral stimuli. Then, the correlations of these individual-level beta values in the significant ROls extracted from LMs with behavioural data were assessed in each of the three contrasts separately. Correlations between pre-and post-scanning craving and dynamic cue-reactivity slopes, between Barratt impulsiveness sum scores and commission error rates and inhibition slopes, and between all four variables and interaction contrast slopes were estimated. The correlation of methamphetamine use duration and all three slopes was also assessed. Lastly, differences in the three slopes in each significant ROI between subjects who hadn't used methamphetamine in the month prior to scanning and those who had were assessed using t-tests. As tests would involve slopes from a number of regions that were dynamically active for each condition, FDR-corrected thresholds of $p<0.05$ were used for these tests.

\section{Results}

\section{Time-invariant activations}

Conventional whole-brain analysis results for the three contrasts can be viewed in Fig. 2. We illustrated whole-brain maps (Z-threshold $=3.1$, corrected cluster-level threshold: $p<0.001$ ) as well as activation changes across the 246 regions using BNA parcellation.

There were higher activations associated with drug vs. neutral cue exposure in the left SFG (superior frontal gyrus) (1734 voxels, peak z-value $=5.82)$, Pcun (precuneus) $(736$ voxels, peak $z$-value $=5.11)$, and lower activations in the right IPL (inferior parietal lobule) $(1326$ voxels, peak $z$-value $=4.55)$ and caudodorsal cingulate cortex (746 voxels, peak $z$-value $=4.95)$, and left caudal-dorsolateral PrG (precentral gyrus) (564 voxels, peak z-value $=4.2)($ Supplementary Table S1). In the inhibition contrast, a cluster in the right medial Pcun (183 voxels, peak z-value $=4.31$ ) survived the threshold, while clusters in the left PrG (1220 voxels, peak z-value $=6.1)$ and right FuG (fusiform gyrus) ( 879 voxels, peak $z$-value $=$ 
5.25), and right IPL (355 voxels, peak z-value $=4.16)$ show a lower activation during inhibition (Supplementary Table S2). The interaction of these two contrasts led to higher activations in the MVOcC (medioventral occipital cortex) (5129 voxels, peak z-value $=6.94)$ and right Pcun (137 voxels, peak zvalue $=4.28$, and lower activations associated with response-inhibition during drug versus neutral cues in the left SFG (352 voxels, peak $z$-value $=4.15)$ and PoG (postcentral gyrus) (1647 voxels, peak z-value $=$ 4.49), and right caudodorsal CG (Cingulate Gyrus) (1317 voxels, peak $z$-value $=4.61$ ) and IPL ( 776 voxels, peak z-value $=4.36)($ Supplementary Table S3).

Whole-brain maps of activations in each of the three windows can be viewed in the supplementary materials (Fig. S1, Fig. S2, and Fig. S3).

\section{Dynamic cue-reactivity}

In the cue-reactivity LME, the main effect of condition (DSNG + DSG or NSNG +NSG) was significant in the medial SFG, sensory thalamus, PCL (paracentral lobule), STG (superior temporal gyrus), rostroventral part of IPL, PoG, hypergranular part of INS (insular gyrus), right caudodorsal CG, MVOcC, and left sensory thalamus (Supplementary Fig.S4). The main effect of time (across three windows) was significant in the right lateral STG $(\mathrm{t}=-5.42, p$-value $=3.0 \mathrm{e}-05)$ and bilateral caudal PhG (parahippocampal gyrus) (left $(\mathrm{t}=-5.1, p$-value $=2.0 \mathrm{e}-04)$, right $(\mathrm{t}=-5.6, p$-value $=2.0 \mathrm{e}-05))$, right rostroposterior STC (superior temporal sulcus) $(\mathrm{t}=-4.7, p$-value $=0.001)$ and bilateral medial Pcun (left $(\mathrm{t}=-5.4, p$-value $=3.0 \mathrm{e}-05)$, right $(\mathrm{t}=-4.9, p$ value $=5.0$ e-04) $)($ Supplementary Fig. S5).

The condition-by-time interaction (condition*time) in the cue-reactivity LME is significant in the right lateral STG $(\mathrm{t}=5.79, p$-value $=5.0 \mathrm{e}-06)$, bilateral rostral STG (left: $\mathrm{t}=4.92, p$-value $=4.0 \mathrm{e}-04$, right: $\mathrm{t}=5.3$, $p$-value $=6.0 \mathrm{e}-05)$ and right ITG (inferior temporal gyrus) $(\mathrm{t}=5.28, p$-value $=6.0 \mathrm{e}-05)$, the left Pcun (dorsomedial parietooccipital sulcus) $(\mathrm{t}=5.4, p$-value $=2.0 \mathrm{e}-04)$, the right medial $(\mathrm{t}=4.69, p$-value $=0.001)$ and lateral amygdala $(\mathrm{t}=5.09, p$-value $=2.0 \mathrm{e}-04)$, and the right rostral hippocampus $(\mathrm{t}=5.38, p$-value $=$ 4.0e-04) (Fig. 3.a). Among these ROIs, only the right rostral STG shows a significant condition effect $(\mathrm{t}=$ $4.16, p$-value $=0.007$ ), all except left rostral STG and right medial amygdala show significant negative main effects of time (Table 2).

Subcortical regions such as the amygdala and hippocampus, as well as the STG and Pcun show a decreasing response over time (habituation) to drug cues compared to neutral cues. (Fig. 4).

\section{Dynamic response-inhibition}

The response-inhibition LME model revealed many regions with significant effect of condition (DSNG + $N S N G$ vs. DSG + NSG) (Supplementary Fig. S4) and significant condition-by-time interactions (Fig. 3.b). The main effect of time was significant in only a handful of regions however, including the bilateral FuG (left $(\mathrm{t}=5.53, p$-value $=2.0 \mathrm{e}-05)$, right $(\mathrm{t}=5.2, p$-value $=1.0 \mathrm{e}-04))$, right medial Pcun $(\mathrm{t}=-4.93, p$-value $=3.0 \mathrm{e}-$ 04), MVOcC and LOcC (lateral occipital cortices) (corrected $p$-value < 0.001 ) (Supplementary Fig. S5). 
Since dynamic inhibition was observed in 107 regions, we only explored the temporal inhibitory and noninhibitory behavior of regions shown to be involved in response-inhibition in a recent meta-analysis of fMRI Go-NoGo tasks ${ }^{53}$. The bilateral MFG (middle frontal gyrus) (left $(\mathrm{t}=5.3, p$-value $=3.7 \mathrm{e}-05)$, right $(\mathrm{t}=$ 4.06, $p$-value $=0.0078)$, right CG $(\mathrm{t}=6.3, p$-value $=6.0 \mathrm{e}-04)$, left INS $(\mathrm{t}=4.3, p$-value $=0.0031)$, right angular gyrus $(\mathrm{t}=7.3, p$-value $=4.0 \mathrm{e}-10)$, right MTG (middle temporal gyrus) $(\mathrm{t}=6.54, p$-value $=7.0 \mathrm{e}-08)$ and left supramarginal gyrus $(\mathrm{t}=4.69, p$-value $=4.0 \mathrm{e}-10)$, showed falling inhibitory activations to NoGo cues and NoGo vs. Go contrast, while the bilateral PrG (left $(\mathrm{t}=-3.58, p$-value $=0.046)$, right $(\mathrm{t}=-4.06, p$ value $=0.0078)$, right SPL $(t=5.97, p$-value $=0.0002)$, and right operculum part of IFG (inferior frontal gyrus) $(\mathrm{t}=-3.68, p$-value $=0.033)$ ) showed increasing activations (sensitization) (Fig. 5).

\section{Table 2}

Regions with significant condition by time interactions in the cue-reactivity and cue-reactivity/responseinhibition interaction linear mixed effects (LME) models. All p-values are FDR-corrected.

\section{Main effect of Cue-reactivity}

(Drug Successful NoGo + Drug Successful Go) $>$ (Neutral Successful NoGo + Neutral Successful Go)

\begin{tabular}{|c|c|c|c|c|c|c|c|c|c|c|c|c|}
\hline \multirow[b]{2}{*}{ Location in BNA } & \multicolumn{4}{|c|}{ Condition } & \multicolumn{4}{|c|}{ Time } & \multicolumn{4}{|c|}{ Condition : Time } \\
\hline & Beta & SE & t-value & $P$-value & Beta & SE & t-value & $P$-value & $\overline{\text { Beta }}$ & SE & t-value & $P$-value \\
\hline Right STG (rostral) & 0.08 & 0.02 & 4.16 & 0.007 & -.0 .06 & 0.01 & -3.81 & 0.04 & 0.13 & 0.02 & 5.3 & 0.000005 \\
\hline Left STG ( rostral ) & 0.03 & 0.01 & 1.92 & 1 & -0.04 & 0.01 & -2.81 & 1 & 0.11 & 0.02 & 4.92 & 0.0004 \\
\hline Right STG (lateral ) & 0.04 & 0.01 & 2.23 & 1 & -.0 .09 & 0.01 & -5.42 & 0.00003 & 0.14 & 0.02 & 5.79 & 0.000006 \\
\hline Right ITG (intermediate ventral) & 0.03 & 0.03 & 0.9 & 1 & -0.13 & 0.03 & -4.4 & 0.0031 & 0.21 & 0.04 & 5.28 & 0.00006 \\
\hline Left Pcun (dmPOS) & 0.01 & 0.03 & 0.44 & 1 & -0.1 & 0.02 & -4.62 & 0.0013 & 0.16 & 0.03 & 5.04 & 0.0000009 \\
\hline Right Amyg (medial) & 0.03 & 0.02 & 1.24 & 1 & -0.04 & 0.02 & -2.44 & 1 & 0.12 & 0.03 & 4.69 & 0.001 \\
\hline Right Amyg (lateral) & 0.03 & 0.02 & 1.53 & 1 & -0.1 & 0.02 & -4.48 & 0.003 & 0.16 & 0.03 & 5.09 & 0.0002 \\
\hline Right Hipp (rostral ) & 0.0009 & 0.03 & 0.04 & 1 & -0.074 & 0.02 & -3.83 & 0.035 & 0.14 & 0.03 & 5.38 & 0.00004 \\
\hline \multicolumn{13}{|c|}{ Cue-reactivity/Inhibition Interaction } \\
\hline \multicolumn{13}{|c|}{ (Drug Successful NoGo + Drug Successful Go) $>$ (Neutral Successful NoGo+ Neutral Successful Go $)$} \\
\hline & \multicolumn{4}{|c|}{ Condition } & \multicolumn{4}{|c|}{ Time } & \multicolumn{4}{|c|}{ Condition : Time } \\
\hline Location in BNA & Beta & SE & t-value & $P$-value & Beta & SE & t-value & $P$-value & Beta & SE & t-value & $P$-value \\
\hline Right Pcun (dmPOS) & -0.06 & 0.02 & -2.51 & 1 & -0.06 & 0.02 & -2.79 & 0.78 & 0.14 & 0.02 & 4.83 & 0.00056 \\
\hline Right PhG (medial) & -0.11 & 0.03 & -3.91 & 0.014 & 0.002 & 0.02 & 0.08 & 1 & 0.19 & 0.03 & 5.59 & 0.000014 \\
\hline Left PhG (medial) & -0.11 & 0.02 & -4 & 0.011 & 0.003 & 0.02 & 0.125 & 1 & 0.2 & 0.03 & 5.75 & 0.000006 \\
\hline
\end{tabular}

Abbreviation: SE: Standard Error, STG: superior temporal gyrus, ITG: inferior temporal gyrus, Pcun (dmPOS): dorsomedial parietooccipital sulcus part of precuneus, Amyg: amygdala, Hipp: hippocampus, PhG: parahippocampal gyrus

\section{Dynamic interaction of cue-reactivity and response- inhibition}


In the LME modeling the interaction of craving and inhibitory processes, the main effect of condition ( $D S N G>D S G$ or $N S N G>N S G$ ) is highly significant across much of the frontal cortex, STG, IPL, Pcun, PoG, dorsal INS, MVOcC, and BG (basal ganglia) (corrected $p$-value < 0.001) (Supplementary Fig. S4). Regions with a significant main effect of time are located in the ITG, FuG, PhG, Pcun, right dorsal and ventral CG, LOcC, and hippocampus (corrected p-value < 0.001) (Supplementary Fig. S5).

The right Pcun show a significant condition-by-time interaction $(\mathrm{t}=4.83, p$-value $=0.0006)$ with an attenuating inhibitory response (habituation) while viewing drug-related cues (DSNG - DSG) but not during neutral cue exposure (NSNG - NSG), and habituation resulted contrast ((DSNG - DSG)> (NSNG - NSG)). The main effects of condition and time are not significant in this region. Bilateral medial PhG also show significant condition-by-time interactions (RPhG: $(\mathrm{t}=5.59, p$-value $=0.00001), \mathrm{LPhG}:(\mathrm{t}=5.75, p$-value $=$ 0.000006)), and show temporally stable activations during response-inhibition in the presence of drugrelated cues but escalating activations during neutral cue exposure (sensitization), and habituation behavior in the interaction of cue-reactivity and inhibition contrast (Fig. 3.c and Fig. 6.a). The main effect of condition is also significant in these regions (RPhG: $(\mathrm{t}=-3.9, p$-value $=0.014), \mathrm{LPhG}:(\mathrm{t}=-4, p$-value $=$ 0.011)) unlike the main effect of time (Table 2).

For the three ROls with a significant condition-by-time interaction in the cue-reactivity/inhibition interaction LME, the temporal behavior of the three contrasts across the overlapping windows is illustrated in Fig. 6.b. The PhG show decreasing estimates (habituation) for the cue-reactivity (DSNG + $D S G>N S N G+N S G$ ) and interaction ( $D S N G-D S G>N S N G-N S G$ ) contrasts, but increasing values of inhibitory control $(D S N G+N S N G>D S G+N S G)$ across time. In the right Pcun, the cue-reactivity contrast and the interaction contrast decrease (sensitization), but the response-inhibition contrast remains mostly stable.

\section{Correlates of dynamic brain activity}

None of the correlations between regional dynamic activation slopes (beta values extracted from the individual-level LMs) and behavioral or clinical variables were significant after FDR correction. There are significant uncorrected correlations between methamphetamine use duration and dynamic cue-reactivity in the right Pcun (beta $=0.28, p$-value $=0.04$ ) and dynamic inhibition in the right LOcC (beta $=0.27, p$ value $=0.044$ ). Dynamic inhibition slopes also had uncorrected correlations with Barratt motor impulsiveness scores in the left PhG (beta $=0.29, p$-value $=0.034$ ) and with commission error rates in the ITG, IPL, Pcun, and dorsal and ventral CG. No significant correlations were observed between slopes derived from individual-level cue-reactivity/inhibition LMs and clinical or behavioral data.

\section{Discussion}

This exploratory study is an investigation of temporally dynamic regional brain activation patterns underlying cue-reactivity, response-inhibition, and their interaction in individuals with MUD. While similar 
sliding window techniques are relatively common in dynamic functional connectivity analyses ${ }^{54,55}$ and despite decades of evidence for temporal variation in regional sensitization and habituation in cognitive/affective neuroscience ${ }^{31-33}$, dynamic analyses of regional activation in addiction remain rare and this is the first study which explored this dynamic interaction in response-inhibition in the context of cue-reactivity.

\section{Dynamic cue-reactivity}

Dynamic cue-reactivity was observed in the bilateral STG, the right amygdala, and rostral hippocampus, and the left Pcun and ITG. Many of these regions have previously been indicated in methamphetamine cue-reactivity $16,17,19$, and drug cue-reactivity more widely ${ }^{56,57}$. Notably, dynamic amygdala activity with a similar downward slope over time has been observed in two recent cue-reactivity studies in individuals with MUD and opioid use disorder ${ }^{37,38}$. A study on individuals with heroin use disorder estimating dynamic causal modeling parameters in overlapping windows has also demonstrated craving inputs to the amygdala increase during a cue-reactivity task, and that the DIPFC's (dorsolateral prefrontal cortex) modulatory impact on the connection between the VMPFC (ventromedial prefrontal cortex) and the amygdala decreases over time ${ }^{58}$. Ekhtiari et al. also similarly reported bilateral dynamic cue-reactivities in the STG, but they observed an initially escalating and subsequently decreasing activation whereas we observed a consistent habituation response ${ }^{37}$. Broadly, our results suggest generalized habituation to drug cues across the task duration.

The dynamic cue-reactivity LME showed no significant condition-by-time interactions in the VMPFC and the VSTR (ventral striatum), indicating a lack of dynamic activity, unlike another dynamic study using similar analytical procedures ${ }^{37}$. Unexpectedly, these regions also showed no static activity, potentially showing that they were not recruited by our task components. Methamphetamine use duration had a significant uncorrected correlation with dynamic cue-reactivity in the right Pcun, similar to a previous study in which addiction severity was found to be correlated with Pcun activation during cue-reactivity tasks ${ }^{59}$.

\section{Dynamic response-inhibition}

More than a hundred regions in our LME model showed dynamic response-inhibitory activity. This may not be surprising, as response-inhibition is associated with large-scale neural activity ${ }^{53}$ and dynamic brain network reconfiguration ${ }^{41}$. Also, notable is that dynamic prefrontal activations were also observed in the response-inhibition model, whereas only FDR-uncorrected prefrontal activations were observed in the other two models (cue-reactivity and cue-reactivity/inhibition interaction). There have been reports of prefrontal sensitization to salient cues ${ }^{36}$, and it has been observed that the prefrontal cortex is implicated in the dysfunctional behavioral regulation seen in the MUD during response control tasks ${ }^{22}$. The 
observation of dynamic activity in prefrontal regions was expected, given their involvement in inhibitory control networks ${ }^{51}$ and response-inhibition in substance use disorders ${ }^{60,61}$.

Most of the regions involved in response-inhibition in a recent meta-analysis of Go-NoGo tasks ${ }^{53}$ had dynamic activation patterns in this study. Notably, while dynamic cue-reactivity was associated with a generalized habituation effect, these regions showed two broad temporal activation patterns. The MTG, the left INS, the right CG, the right MTG, and supramarginal gyrus, showed falling inhibitory activations while the PrG, the left SPL, and IFG (operculum) showed increasing activations (sensitization). This might reflect differences in response-inhibitory processes that these regions contribute to, such as error monitoring and attentional control ${ }^{62,63}$, or the involvement of these regions in other networks that interact with the response-inhibition network in individuals with substance use disorders, such as the INS in the salience network or the MFG in self-directed processing ${ }^{14}$.

Commission error rates have been used as a measure of response-inhibitory success in Go-NoGo tasks, and have been correlated with activities in the right SPL and DLPFC in individuals with addictive disorders ${ }^{64}$. In our study, commission error rates had significant uncorrected correlations with dynamic response-inhibition slopes commission error rates in the ITG, IPL, Pcun, and dorsal and ventral anterior CG, potentially implicating these regions in response-inhibition dysfunctions in the MUD. These regions can play an important role in the development of response control-related biomarkers in addictive disorders ${ }^{65}$.

\section{Dynamic response-inhibition during cue exposure}

The bilateral PhG and the right Pcun were the only regions with a dynamic interaction of responseinhibition and cue-reactivity. Several meta-analyses have demonstrated that drug cue-reactivity is associated with heightened precuneal activation ${ }^{66,67}$, and based on the response-inhibition literature, dopaminergic inhibition and network decoupling of precuneal activity may be important for successful response-inhibition ${ }^{68-70}$. Precuneal involvement in cue-reactivity in substance use disorders might be related to its role in the default mode network and self-referential processing in general ${ }^{14}$, and, interestingly, it has been argued that the Pcun might be an important node for the integration of contradictory executive control and cue-reactivity processes ${ }^{71}$. Considering the above, the decreasing activation associated with drug-related inhibition in the right Pcun may reflect a lessening effect of drug cues in hampering response-inhibition across the task duration. Since it appears that the responseinhibition contrast in the Pcun is mostly stable across time while cue-reactivity and interaction contrasts decline, habituation to drug cues or top-down suppression of precuneal cue-reactivity, rather than the role of the Pcun in response-inhibition per se, maybe the responsible mechanisms.

The PhG have also been implicated in substance use disorders. Addictive disorders are associated with parahippocampal gray matter changes ${ }^{72}$ and increases in its connectivity within the default mode network ${ }^{73}$, both the right and the left parahippocampus generally show higher activations in response to 
drug-related cues compared to neutral cues $66,74,75$, and response-inhibition-associated parahippocampal dysfunction has been observed in individuals with substance use disorders compared to healthy controls 20. As part of the default mode network and given its association with drug cue-reactivity, it was expected that similar to the Pcun, the cue-reactivity contrast in the PhG would decrease, reflecting both habituation processes and task-engagement-related suppression. Some evidence also exists for parahippocampal habituation during exposure to emotionally salient stimuli 76,77 and for the role of the parahippocampus in the extinguishing of drug cue associations ${ }^{78}$. However, the parahippocampus is also involved in neural networks involved in associational memory and learning ${ }^{79,80}$ and might be activated to support learning during response-inhibition tasks ${ }^{14}$. Indeed, increasing parahippocampal recruitment during a learning task has been observed before ${ }^{81}$. These dual roles of the parahippocampus in cue habituation and learning could explain why the cue-reactivity contrast decreased while the response-inhibition contrast increased in the PhG during the task, and is supported by the observation that drug-related inhibition remained mostly stable, while inhibition during neutral cue exposure was associated with increasing parahippocampal activity.

An interesting observation in this study was the right-lateralization of dynamically active regions across the three contrasts. Some evidence exists that the right hemisphere may be more important in responseinhibitory and attentional control processes ${ }^{82,83}$, and right lateralization of dynamic response to salient stimuli has been observed in the right amygdala, IPL, and hippocampus ${ }^{31,84}$. It has been argued that while the left amygdala is involved in sustained stimulus evaluation, the right amygdala might be more specialized for dynamic stimulus processing ${ }^{34}$.

\section{Limitations}

While the results of this exploratory investigation are promising, several limitations are important to point out. Firstly, we included no healthy control group, and so the specificity of observed patterns to individuals with MUD is unclear. Also, all participants were men, treatment seeking individuals MUD, limiting the generalizability of our observations. Regarding the task design, an inherent limitation introduced by our use of a mixed drug cue and negative emotional Go-NoGo task is the potential carryover effects of salient cues on brain activity during subsequent blocks ${ }^{85,86}$. While such issues may be ameliorated by the choice of a blocked presentation of different cue types, the results are likely confounded by these effects. Lastly, we used no measure of craving across the task duration, which would have allowed the analysis of temporal correlations between craving and neural activity, as in one recent study by Murphy et al. ${ }^{38}$.

\section{Conclusion}

This study provides preliminary evidence that a mixed event-block Go-NoGo/cue-reactivity task can be used to assess the temporal dynamics of cue-reactivity, response-inhibition, and their interaction. The 
regions with a temporally dynamic response are involved in various neuro-cognitive aspects of addictive disorders. Notably, we observed dynamic amygdalar activity in both response-inhibition and cue-reactivity contrasts, and there is extensive literature on the time-variance of amygdalar activity $31,34,37,87$, and it has been recently argued that amygdalar habituation is a more reliable index than mean amplitude ${ }^{88}$. The interaction of cue-reactivity and response-inhibition occurred in regions in which the neural activations associated with cue-reactivity and response-inhibition followed broadly opposing slopes across time, namely in the parahippocampal regions and the precuneus, suggesting that these regions may be important hubs where response-inhibitory and cue-reactivity processes integrate. Dynamic interactions in these regions may help biomarker development and suggest new targets for interventions, and it has been suggested that failures to inhibit precuneal cue-reactivity may predict relapse ${ }^{89}$, and impairments of parahippocampal habituation are associated with poorer treatment outcomes in cocaine users ${ }^{39}$. Future studies could make use of better power analyses, flexible sliding window sizes and inference methods, prospective designs, and replication across different populations and time-points to assess the stability, generalizability, and potential predictive utility of these dynamic activation and interaction parameters.

\section{Declarations}

\section{Funding}

This study was founded by Tehran University of Medical Sciences and was also reviewed and partially supported by the Cognitive Sciences and Technologies Council (CSTC) under the grant numbers 9336 and 8651 .

\section{Acknowledgment}

Authors would like to thank Ashkan Faghiri and Seyed Amirhossein Batouli for their great work in the data collection for this project.

\section{Data availability}

The raw database for this study is available on reasonable request to the corresponding authors.

\section{Code availability}

$\mathrm{R}$ codes and beta values of this study are available on request to the corresponding authors.

\section{Author contributions}


S.J., H.E., K.K and A.S. designed the study. H.E., A.D., and M.A.O. collected the data. S.J. performed simulations and data analysis under H.E., K.K., A.A., and M.S.H. supervision. S.J. and A.S wrote the paper with input from H.E. K.K., A.A., M.S.H., M.A.O., and A.D. All authors (S.J., A.S., A.A., M.S.H., A.D., M.A.O., K.K and H.E.) contributed in manuscript preparation.

All authors (S.J., A.S., A.A., M.S.H., M.A.O., A.D., K.K and H.E.) agreed on the final manuscript before submission

\section{Competing interests}

The authors declare no competing interests.

\section{References}

1. United Nations Office on Drugs and Crime. World Drug Report 2020.United Nations Publications. Retrieved from // wdr.unodc.org/wdr2020/en/index.html (2020).

2. Substance Abuse and Mental Health Services Administration. Key Substance Use and Mental Health Indicators in the United States: Results from the 2019 National Survey on Drug Use and Health. Retrieved from https://www.samhsa.gov/data/ (2020).

3. Hedegaard, H., Bastian, B. A., Trinidad, J. P., Spencer, M. \& Warner, M. Drugs Most Frequently Involved in Drug Overdose Deaths: United States, 2011-2016. Natl Vital Stat Rep, 67, 1-14 (2018).

4. Mendelson, J., Baggott, M. J., Flower, K. \& Galloway, G. Developing Biomarkers for Methamphetamine Addiction. Current neuropharmacology, 9 (1), 100-103 (2011).

5. Paulus, M. Methamphetamine use disorder: Epidemiology, clinical manifestations, course, assessment, and diagnosis. UpToDate. Saxon AJ (Ed.)(2018).

6. Gouzoulis-Mayfrank, E. et al. Methamphetamine-related disorders. Deutsches Ärzteblatt International, 114 (26), 455 (2017).

7. Paulus, M. P., Stewart, J. L. \& Neurobiology Clinical Presentation, and Treatment of Methamphetamine Use Disorder: A Review. JAMA Psychiatry, 77 (9), 959-966 (2020).

8. Potvin, S. et al. Cognitive deficits in individuals with methamphetamine use disorder: A metaanalysis. Addict behaviors, 80, 154-160 (2018).

9. Jan, R. K., Kydd, R. R. \& Russell, B. R. Functional and Structural Brain Changes Associated with Methamphetamine Abuse. Brain Sciences, 2 (4), 434-482 (2012).

10. Stewart, J. L. et al. Striatum and insula dysfunction during reinforcement learning differentiates abstinent and relapsed methamphetamine-dependent individuals., 109 (3), 460-471 (2014).

11. Ekhtiari, H., Nasseri, P., Yavari, F., Mokri, A. \& Monterosso, J. Neuroscience of drug craving for addiction medicine: From circuits to therapies. Prog. Brain Res, 223, 115-141 (2016). 
12. Seow, L. S. E., Ong, W. J., Hombali, A., AshaRani, P. V. \& Subramaniam, M. A Scoping Review on Cue Reactivity in Methamphetamine Use Disorder. Int J Environ Res Public Health, 17 (18), 6540 (2020).

13. Monterosso, J. R., Aron, A. R., Cordova, X., Xu, J. \& London, E. D. Deficits in response inhibition associated with chronic methamphetamine abuse. Drug and Alcohol Dependence, 79 (2), 273-277 (2005).

14. Zilverstand, A., Huang, A. S., Alia-Klein, N. \& Goldstein, R. Z. Neuroimaging Impaired Response Inhibition and Salience Attribution in Human Drug Addiction: A Systematic Review., 98 (5), 886-903 (2018).

15. Lannoy, S. et al. A dual-process exploration of binge drinking: Evidence through behavioral and electrophysiological findings. Addict Biol, 25 (2), e12685 (2020).

16. Grodin, E. N., Courtney, K. E. \& Ray, L. A. Drug-Induced Craving for Methamphetamine Is Associated With Neural Methamphetamine Cue Reactivity. J Stud Alcohol Drugs, 80 (2), 245-251 (2019).

17. Huang, S. et al. Craving responses to methamphetamine and sexual visual cues in individuals with methamphetamine use disorder after long-term drug rehabilitation. Front. Psychiatry, 9, 145 (2018).

18. MacNiven, K. H. et al. Association of neural responses to drug cues with subsequent relapse to stimulant use. JAMA network open, 1 (8), e186466-e186466 (2018).

19. Malcolm, R. et al. Regional Brain Activity in Abstinent Methamphetamine Dependent Males Following Cue Exposure.J Drug Abuse2(1), (2016).

20. Harlé, K. M., Zhang, S., Ma, N., Yu, A. J. \& Paulus, M. P. Reduced Neural Recruitment for Bayesian Adjustment of Inhibitory Control in Methamphetamine Dependence. Biological Psychiatry: Cognitive Neuroscience and Neuroimaging, 1 (5), 448-459 (2016).

21. Nestor, L. J., Ghahremani, D. G., Monterosso, J. \& London, E. D. Prefrontal hypoactivation during cognitive control in early abstinent methamphetamine-dependent subjects. Psychiatry Research: Neuroimaging, 194 (3), 287-295 (2011).

22. Salo, R., Fassbender, C., Buonocore, M. H. \& Ursu, S. Behavioral regulation in methamphetamine abusers: An fMRI study. Psychiatry Research: Neuroimaging, 211 (3), 234-238 (2013).

23. He, X., Zhao, D., Zhang, M., Leng, Y. \& He, W. Cue Exposure Triggers Inhibitory Deficits in Individuals With Methamphetamine Use Disorder. J. Stud. Alcohol Drugs, 82 (2), 197-203 (2021).

24. Noël, X. et al. Alcohol cues increase cognitive impulsivity in individuals with alcoholism., 192 (2), 291-298 (2007).

25. Weafer, J. \& Fillmore, M. T. Alcohol-related stimuli reduce inhibitory control of behavior in drinkers., 222 (3), 489-498 (2012).

26. Papachristou, H., Nederkoorn, C., Havermans, R., van der Horst, M. \& Jansen, A. Can't stop the craving: The effect of impulsivity on cue-elicited craving for alcohol in heavy and light social drinkers., 219 (2), 511-518 (2012).

27. Gilman, J. M. et al. Anterior insula activation during inhibition to smoking cues is associated with ability to maintain tobacco abstinence. Addictive Behaviors Reports, 7, 40-46 (2018). 
28. Stein, M. et al. Alcohol-related context modulates neural correlates of inhibitory control in alcohol dependent patients: Preliminary data from an fMRI study using an alcohol-related Go/NoGo-task. Behav. Brain. Res, 398, 112973 (2021).

29. Ames, S. L. et al. Neural correlates of a Go/NoGo task with alcohol stimuli in light and heavy young drinkers. Behav. Brain Res, 274, 382-389 (2014).

30. Czapla, M. et al. Do alcohol-dependent patients show different neural activation during response inhibition than healthy controls in an alcohol-related fMRI go/no-go-task?, 234 (6), 1001-1015 (2017).

31. Fischer, $\mathrm{H}$. et al. Brain habituation during repeated exposure to fearful and neutral faces: A functional MRI study. Brain Res. Bull, 59 (5), 387-392 (2003).

32. Moses-Kolko, E. L. et al. Rapid habituation of ventral striatal response to reward receipt in postpartum depression. Biol. Psychiatry, 70 (4), 395-399 (2011).

33. Phan, K. L., Liberzon, I., Welsh, R. C., Britton, J. C. \& Taylor, S. F. Habituation of rostral anterior cingulate cortex to repeated emotionally salient pictures. Neuropsychopharmacology, 28 (7), 13441350 (2003).

34. Wright, C. I. et al. Differential prefrontal cortex and amygdala habituation to repeatedly presented emotional stimuli., 12 (2), 379-383 (2001).

35. Quirk, G. J. \& Mueller, D. Neural Mechanisms of Extinction Learning and Retrieval. Neuropsychopharmacology, 33 (1), 56-72 (2008).

36. Strauss, M. M. et al. fMRI of sensitization to angry faces. Neurolmage, 26 (2), 389-413 (2005).

37. Ekhtiari, H., Kuplicki, R., Aupperle, R. L. \& Paulus, M. P. It is Never as Good the Second Time Around: Brain Areas Involved in Salience Processing Habituate During Repeated Drug Cue Exposure in Treatment Engaged Abstinent Methamphetamine and Opioid Users. Neurolmage, 238, 118180 https://doi.org/10.1016/j.neuroimage.2021.118180 (2021).

38. Murphy, A. et al. Time-dependent neuronal changes associated with craving in opioid dependence: an fMRI study. Addict Biol. 23(5),1168-1178(2017) doi:10.1111/adb.12554.

39. Regier, P. S. et al. Sustained brain response to repeated drug cues is associated with poor drug-use outcomes.Addiction biologye13028(2021).

40. Nakata, H., Sakamoto, K., Honda, Y. \& Kakigi, R. Temporal dynamics of neural activity in motor execution and inhibition processing. Eur J Neurosci, 41 (11), 1448-1458 (2015).

41. Wang, B. et al. Dynamic reconfiguration of functional brain networks supporting response inhibition in a stop-signal task. Brain Imaging and Behavior, 14 (6), 2500-2511 (2020).

42. American Psychiatric Association. Diagnostic and Statistical Manual of Mental Disorders, 4th Edition, Text Revision (DSM-IV-TR). (American Psychiatric Association, 2000).

43. Oldfield, R. C. The assessment and analysis of handedness: The Edinburgh inventory., 9 (1), 97-113 (1971). 
44. Barratt, E. S. Impulsiveness and Aggression From Violence and Mental Disorder: Developments in Risk Assessment61-79(The University of Chicago Press, 1994).

45. Osman, A. et al. The Depression Anxiety Stress Scales-21 (DASS-21): Further Examination of Dimensions, Scale Reliability, and Correlates. Journal of Clinical Psychology, 68 (12), 1322-1338 (2012).

46. Crawford, J. R. \& Henry, J. D. The positive and negative affect schedule (PANAS): construct validity, measurement properties and normative data in a large non-clinical sample. Br J Clin Psychol, 43 (3), 245-265 (2004).

47. Ekhtiari, H., Alam-Mehrjerdi, Z., Nouri, M., George, S. \& Mokri, A. Designing and evaluation of reliability and validity of visual cue-induced craving assessment task for methamphetamine smokers. Basic and Clinical Neuroscience, 1 (4), 34-37 (2010).

48. Lang, P. J., Bradley, M. M. \& Cuthbert, B. N. International affective picture system (IAPS): Technical manual and affective ratings. NIMH Center for the Study of Emotion and Attention, 1 (39-58), 3 (1997).

49. Jenkinson, M., Beckmann, C. F., Behrens, T. E., Woolrich, M. W. \& Smith, S. M. Fsl. Neuroimage, 62 (2), 782-790 (2012).

50. Hallquist, M. N., Hwang, K. \& Luna, B. The nuisance of nuisance regression: spectral misspecification in a common approach to resting-state fMRI preprocessing reintroduces noise and obscures functional connectivity., 82, 208-225 (2013).

51. Fan, L. et al. The human brainnetome atlas: a new brain atlas based on connectional architecture. Cerebral cortex, 26 (8), 3508-3526 (2016).

52. Team, R. C. R: A language and environment for statistical computing(2013).

53. Zhang, R., Geng, X. \& Lee, T. M. C. Large-scale functional neural network correlates of response inhibition: an fMRI meta-analysis. Brain Struct Funct, 222 (9), 3973-3990 (2017).

54. Sakoglu, U. et al. Classification of cocaine-dependent participants with dynamic functional connectivity from functional magnetic resonance imaging data. J Neurosci Res, 97 (7), 790-803 (2019).

55. Vergara, V. M., Weiland, B. J., Hutchison, K. E. \& Calhoun, V. D. The Impact of Combinations of Alcohol, Nicotine, and Cannabis on Dynamic Brain Connectivity. Neuropsychopharmacol, 43 (4), 877-890 (2018).

56. Chase, H. W., Eickhoff, S. B., Laird, A. R. \& Hogarth, L. The Neural Basis of Drug Stimulus Processing and Craving: An Activation Likelihood Estimation Meta-Analysis. Biol. Psychiatry, 70 (8), 785-793 (2011).

57. Kühn, S. \& Gallinat, J. Common biology of craving across legal and illegal drugs - a quantitative meta-analysis of cue-reactivity brain response. Eur. J. Neurosci, 33 (7), 1318-1326 (2011).

58. Zare-Sadeghi, A., Oghabian, M. A., Zare-Bidoky, M., Batouli, S. A. H. \& Ekhtiari, H. How Top-Down and Bottom-Up Regulation in Fronto-Amygdalar Network Changes over Time during Drug Cue-Exposure: An fMRI Study among Abstinent Heroin User. bioRxiv 678961, doi:10.1101/678961 (2019). 
59. Courtney, K. E., Ghahremani, D. G., London, E. D. \& Ray, L. A. The association between cue-reactivity in the precuneus and level of dependence on nicotine and alcohol. Drug Alcohol Depend, 141, 21-26 (2014).

60. Goldstein, R. Z. \& Volkow, N. D. Dysfunction of the prefrontal cortex in addiction: neuroimaging findings and clinical implications. Nat Rev Neurosci, 12 (11), 652-669 (2011).

61. Qiu, Z. \& Wang, J. Altered neural activities during response inhibition in adults with addiction: a voxelwise meta-analysis. Psychol. Med, 51 (3), 387-399 (2021).

62. Luijten, M. et al. Systematic review of ERP and fMRI studies investigating inhibitory control and error processing in people with substance dependence and behavioural addictions. J Psychiatry Neurosci, 39, 149-169 (2014).

63. Tiego, J., Testa, R., Bellgrove, M. A., Pantelis, C. \& Whittle S. A hierarchical model of inhibitory control. Front. Psychol, 9, 1339 (2018).

64. Liu, G. C. et al. Brain activation for response inhibition under gaming cue distraction in internet gaming disorder. Kaohsiung J. Med. Sci, 30 (1), 43-51 (2014).

65. Moeller, S. J. \& Paulus, M. P. Toward biomarkers of the addicted human brain: Using neuroimaging to predict relapse and sustained abstinence in substance use disorder. Progress in NeuroPsychopharmacology and Biological Psychiatry, 80, 143-154 (2018).

66. Engelmann, J. M. et al. Neural substrates of smoking cue reactivity: A meta-analysis of fMRI studies. Neurolmage, 60 (1), 252-262 (2012).

67. Schacht, J. P., Anton, R. F. \& Myrick, H. Functional neuroimaging studies of alcohol cue reactivity: a quantitative meta-analysis and systematic review. Addict Biol, 18 (1), 121-133 (2013).

68. Albrecht, D. S., Kareken, D. A., Christian, B. T., Dzemidzic, M. \& Yoder, K. K. Cortical Dopamine Release During a Behavioral Response Inhibition Task. Synapse, 68 (6), 266-274 (2014).

69. Tomasi, D. et al. Dopamine transporters in striatum correlate with deactivation in the default mode network during visuospatial attention. PLoS One, 4 (6), (2009). e6102

70. van Rooij, D. et al. Altered neural connectivity during response inhibition in adolescents with attention-deficit/hyperactivity disorder and their unaffected siblings. Neuroimage Clin, 7, 325-335 (2015).

71. Dong, G. H. et al. Addiction severity modulates the precuneus involvement in internet gaming disorder: Functionality, morphology and effective connectivity. Progress in NeuroPsychopharmacology and Biological Psychiatry, 98, 109829 (2020).

72. Van Dam, N. T., Rando, K., Potenza, M. N., Tuit, K. \& Sinha, R. Childhood maltreatment, altered limbic neurobiology, and substance use relapse severity via trauma-specific reductions in limbic gray matter volume. JAMA Psychiatry, 71 (8), 917-925 (2014).

73. Wang, Y. et al. Abnormal Functional Connectivity in Cognitive Control Network, Default Mode Network, and Visual Attention Network in Internet Addiction: A Resting-State fMRI Study. Front. Neurol, 10, 1006 (2019). 
74. Noori, H. R., Cosa Linan, A. \& Spanagel, R. Largely overlapping neuronal substrates of reactivity to drug, gambling, food and sexual cues: A comprehensive meta-analysis. Eur Neuropsychopharmacol, 26 (9), 1419-1430 (2016).

75. Tang, D. W., Fellows, L. K., Small, D. M. \& Dagher, A. Food and drug cues activate similar brain regions: a meta-analysis of functional MRI studies. Physiol. Behav, 106 (3), 317-324 (2012).

76. Denny, B. T. et al. Insula-amygdala functional connectivity is correlated with habituation to repeated negative images. Social Cognitive and Affective Neuroscience, 9 (11), 1660-1667 (2014).

77. Feinstein, J. S., Goldin, P. R., Stein, M. B., Brown, G. G. \& Paulus, M. P. Habituation of attentional networks during emotion processing., 13 (10), 1255-1258 (2002).

78. Konova, A. B. et al. Neural mechanisms of extinguishing drug and pleasant cue associations in human addiction: role of the VMPFC. Addict Biol, 24 (1), 88-99 (2019).

79. Davachi, L. Item, context and relational episodic encoding in humans. Curr Opin Neurobiol, 16 (6), 693-700 (2006).

80. Ritchey, M., Libby, L. A. \& Ranganath, C. Cortico-hippocampal systems involved in memory and cognition: the PMAT framework. Prog Brain Res, 219, 45-64 (2015).

81. Blest-Hopley, G., O’Neill, A., Wilson, R., Giampietro, V. \& Bhattacharyya, S. Disrupted parahippocampal and midbrain function underlie slower verbal learning in adolescent-onset regular cannabis use., 238 (5), 1315-1331 (2021).

82. D'Alberto, N., Funnell, M., Potter, A. \& Garavan, H. A split-brain case study on the hemispheric lateralization of inhibitory control., 99, 24-29 (2017).

83. Hampshire, A., Chamberlain, S. R., Monti, M. M., Duncan, J. \& Owen, A. M. The role of the right inferior frontal gyrus: inhibition and attentional control., 50 (3), 1313-1319 (2010).

84. Sun, Y. et al. Brain fMRI study of crave induced by cue pictures in online game addicts (male adolescents). Behav. Brain Res, 233 (2), 563-576 (2012).

85. Sayette, M. A., Griffin, K. M. \& Sayers, W. M. Counterbalancing in Smoking Cue Research: A Critical Analysis. Nicotine Tob Res, 12 (11), 1068-1079 (2010).

86. Wilson, S. J., Sayette, M. A., Fiez, J. A. \& Brough, E. Carry-over effects of smoking cue exposure on working memory performance. Nicotine Tob Res, 9 (5), 613-619 (2007).

87. Yin, S., Liu, Y., Petro, N. M., Keil, A. \& Ding, M. Amygdala adaptation and temporal dynamics of the salience network in conditioned fear: a single-trial fMRI study. eNeuro 5(1)(2018).

88. Plichta, M. M. et al. Amygdala habituation: A reliable fMRI phenotype. Neurolmage, 103, 383-390 (2014).

89. Versace, F. et al. Prequit $\mathrm{fMRI}$ responses to pleasant cues and cigarette-related cues predict smoking cessation outcome. Nicotine Tob. Res, 16 (6), 697-708 (2014).

\section{Figures}



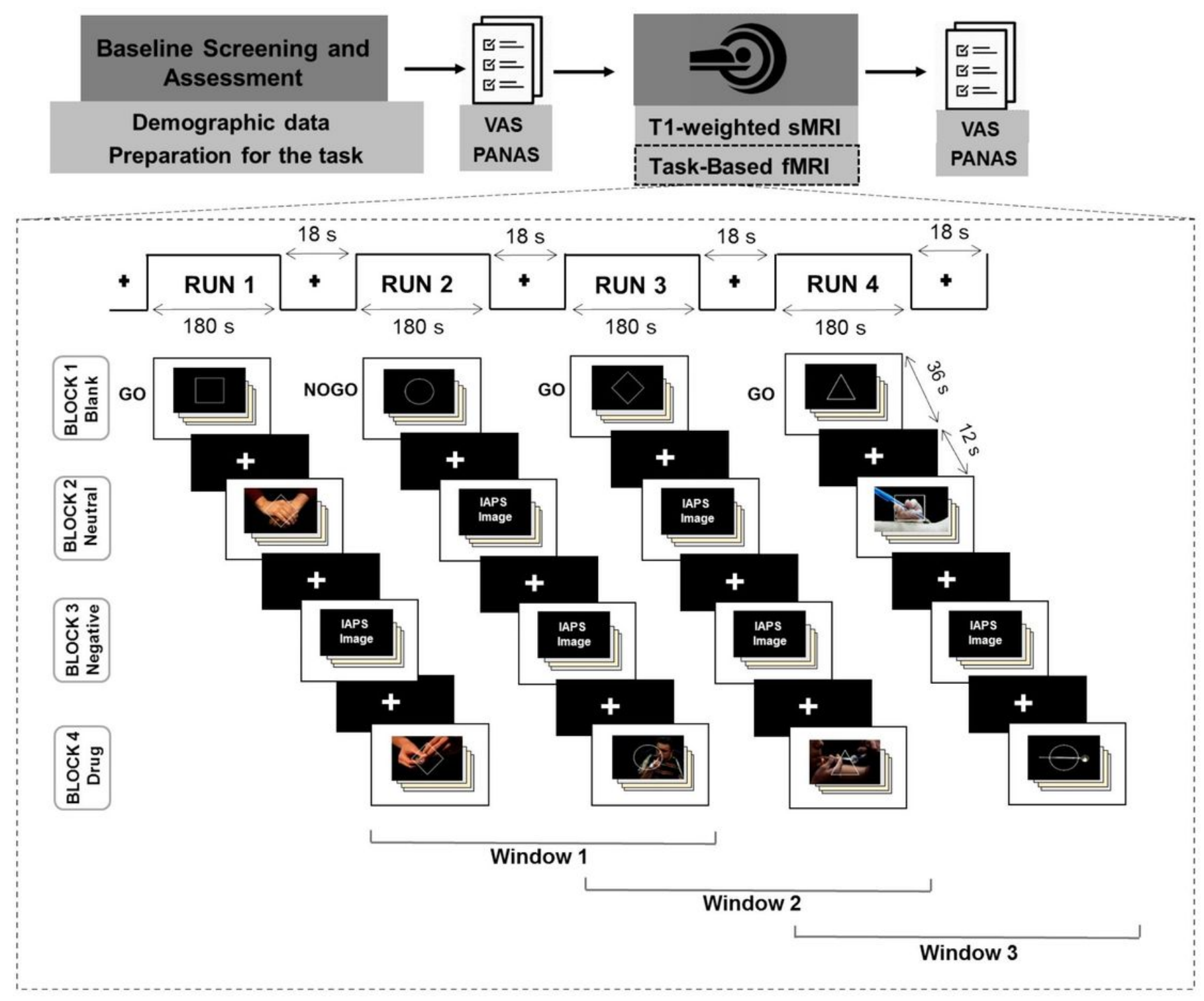

Figure 1

fMRI task structure and timing: The task contains four runs, each run contains four blocks with Go-NoGo stimuli superimposed over different backgrounds: Blank, Drug, Neutral, and Negative emotional stimuli (180 seconds). The blocks are separated by resting blocks with fixation points (18 seconds). In all blocks, the Go stimuli are triangle, square, and diamond, and the NoGo stimulus is a circle. Negative pictures and some neutral pictures were taken from the IAPS database. Note that none of the utilized IAPS pictures are displayed here due to the copyright limitations. Drug pictures and some neutral pictures taken from other databases with copyright permission are shown here. Abbreviation: VAS: Visual Analog Scale, PANAS: Positive and Negative Scale. 


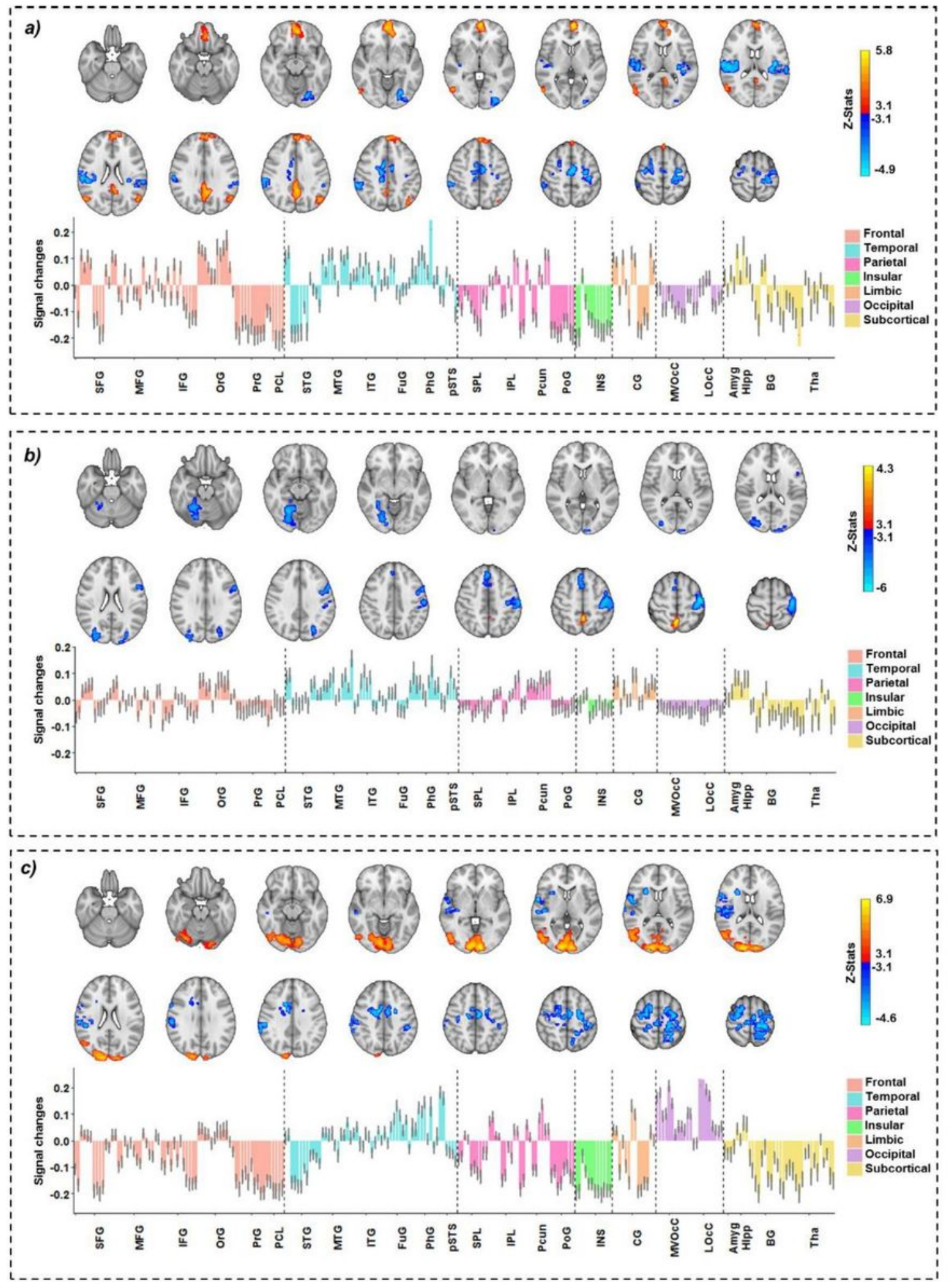

\section{Figure 2}

Whole-brain response to the task-based fMRI in three contrasts. Brain activation maps (Z-threshold=3.1, corrected cluster-level threshold: $p<0.001)$ and changes in brain activation in Brainnetome (BNA) regions. The colored bars show mean parameter estimates, and the error bars show the standard error of zstatistic values across 53 methamphetamine use disorders (MUD). (a) The main effect of cue-reactivity ((Drug Successful NoGo+Drug Successful Go) > (Neutral Successful NoGo + Neutral Successful Go)). (b) 
The main effect of inhibition ((Drug Successful NoGo+Neutral Successful NoGo) > (Drug Successful Go+Neutral Successful Go)). (c) The main effect of response-inhibition during cue-reactivity ((Drug Successful NoGo>Drug Successful Go) > (Neutral Successful NoGo>Neutral Successful Go)).

Abbreviation: SFG: superior frontal gyrus, MFG: middle frontal gyrus, IFG: inferior frontal gyrus, OrG: orbital gyrus, PrG: precentral gyrus, PCL: paracentral lobule, STG: superior temporal Gyrus, MTG: middle temporal gyrus, ITG: inferior temporal gyrus, FuG: fusiform gyrus, PhG: parahippocampal gyrus, pSTS:: posterior superior temporal sulcus, SPL: superior parietal lobule, IPL: inferior parietal lobule, Pcun: precuneus, PoG: postcentral gyrus, INS: insular gyrus, CG: cingulate gyrus, MVOcC: medioventral occipital cortex, LOcC: lateral occipital cortex, Amyg: amygdala, Hipp: hippocampus, BG: basal ganglia, Tha: thalamus. 


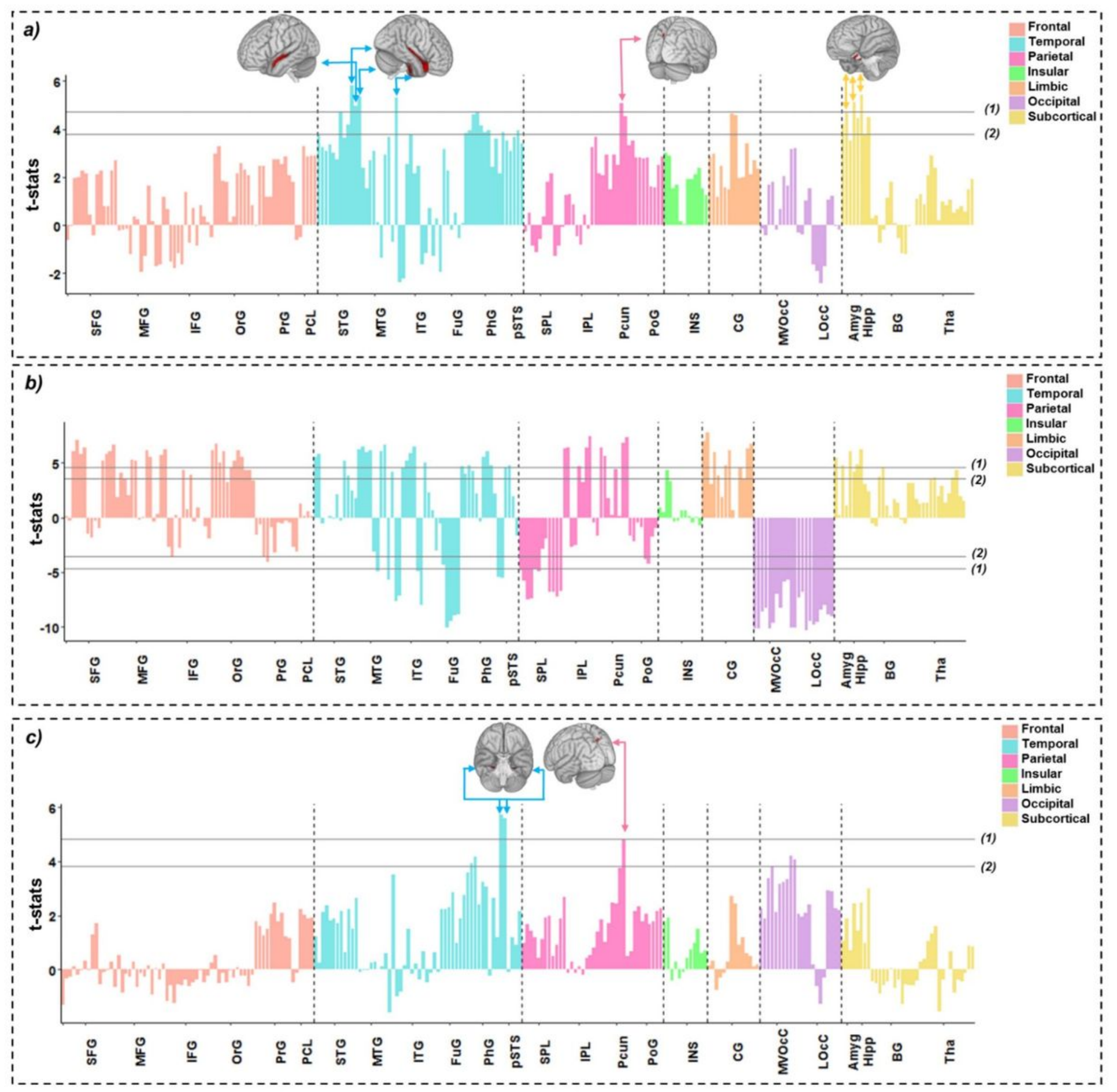

\section{Figure 3}

Condition by time interactions in the three linear mixed effects (LME) models. Bars show the coefficient of the condition by time (three windows) interaction term in the three LMEs (with condition*time as a fixed effect and subjects as a random effect) for each Brainnetome (BNA) subregion. 3D brains are those with significant activations in whole brain analyses. (a) Coefficient of interaction in the cue-reactivity LME ((Drug Successful NoGo+Drug Successful Go) or (Neutral Successful NoGo+Neutral Successful Go)). (b) Coefficient of interaction in the response-inhibition LME ((Drug Successful NoGo+Neutral Successful NoGo) or (Drug Successful Go+Neutral Successful Go)). (c) Coefficient of interaction in the cue- 
reactivity/response-inhibition interaction LME ((Drug Successful NoGo>Drug Successful Go) or (Neutral Successful NoGo>Neutral Successful Go)). (1) FDR corrected p-value<0.001. (2) FDR corrected pvalue $<0.05$. Abbreviation: SFG: superior frontal gyrus, MFG: middle frontal gyrus, IFG: inferior frontal gyrus, OrG: orbital gyrus, PrG: precentral gyrus, PCL: paracentral lobule, STG: superior temporal Gyrus, MTG: middle temporal gyrus, ITG: inferior temporal gyrus, FuG: fusiform gyrus, PhG: parahippocampal gyrus, pSTS:: posterior superior temporal sulcus, SPL: superior parietal lobule, IPL: inferior parietal lobule, Pcun: precuneus, PoG: postcentral gyrus, INS: insular gyrus, CG: cingulate gyrus, MVOcC: medioventral occipital cortex, LOcC: lateral occipital cortex, Amyg: amygdala, Hipp: hippocampus, BG: basal ganglia, Tha: thalamus. 


\section{Conditions}

\section{- Cue-reactivity Contrast \\ - - Drug Successful NoGo + Drug Successful Go \\ - Neutral Successful NoGo + Neutral Successful Go}
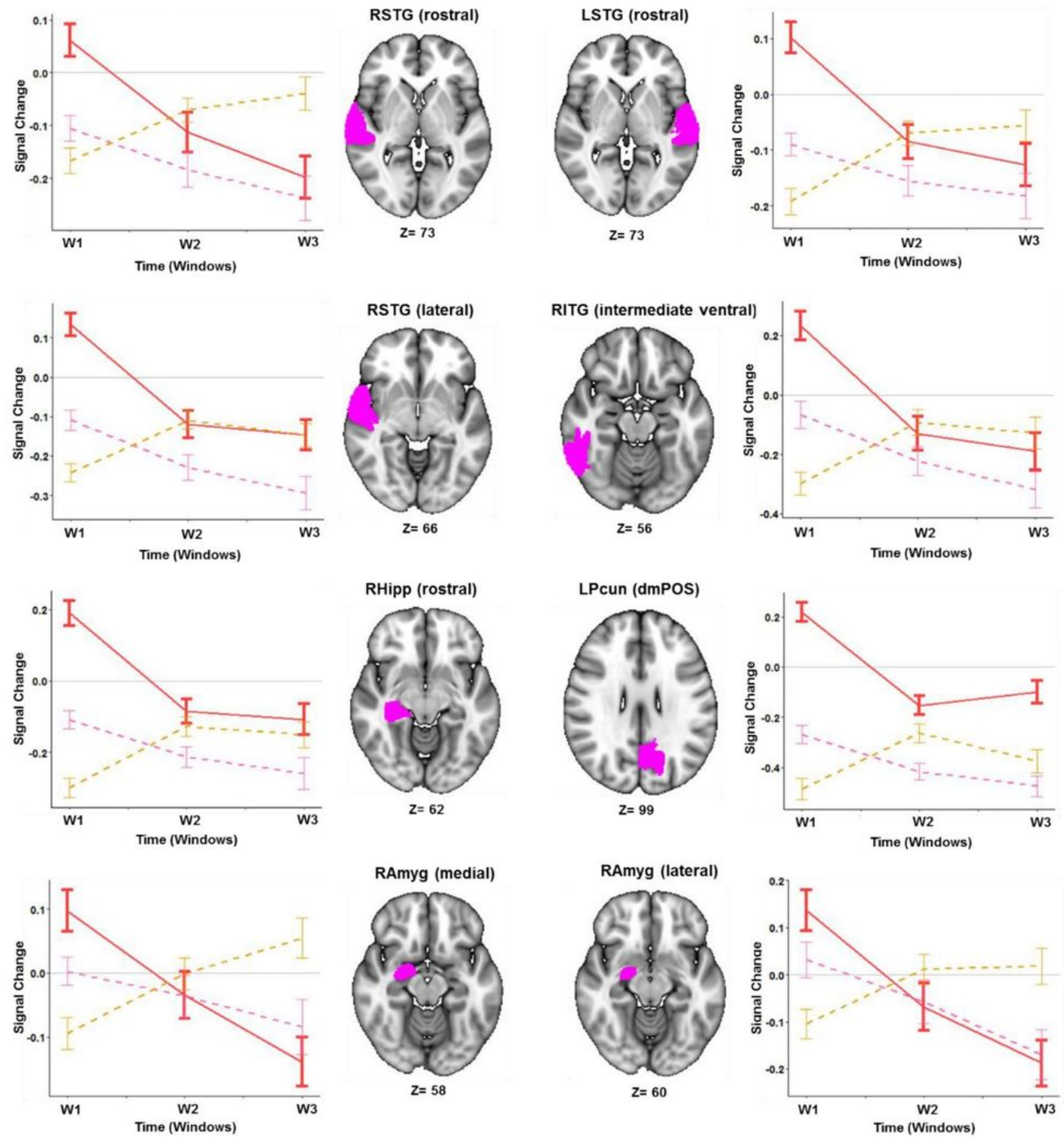

Figure 4

Temporal behavior of ROls with significant condition by time interactions in the cue-reactivity linear mixed effects (LME) model. Lines show the average main effect of Drug (Drug Successful NoGo+Drug Successful Go) or Neutral (Neutral Successful NoGo+Neutral Successful Go) cue-exposure condition in the Brainnetome (BNA) regions or the result of Drug vs. Neutral contrast, and error bars show the standard error of z-statistic values across 53 methamphetamine use disorders (MUD) at each temporal 
window (FDR corrected p-value<0.001). Abbreviation: RSTG: right superior temporal gyrus, LSTG: left superior temporal gyrus, RITG: right inferior temporal gyrus, RHipp: right hippocampus, LPcun: left precuneus, RAmyg: right amygdala.

\section{Conditions}

- Inhibition Contrast

- - Drug Successful NoGo + Neutral Successful NoGo

- - Drug Successful Go + Neutral Successful Go
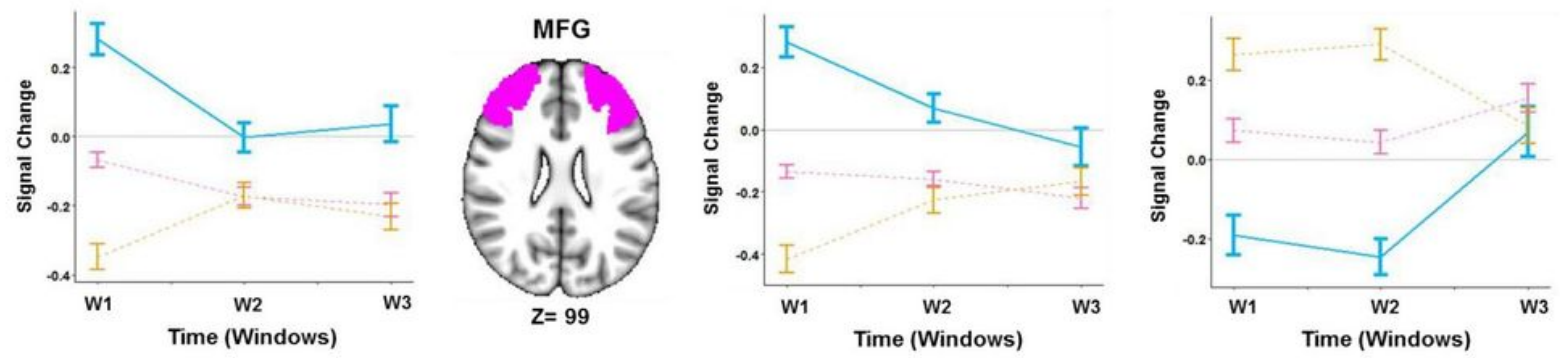

RIFG (opercular)
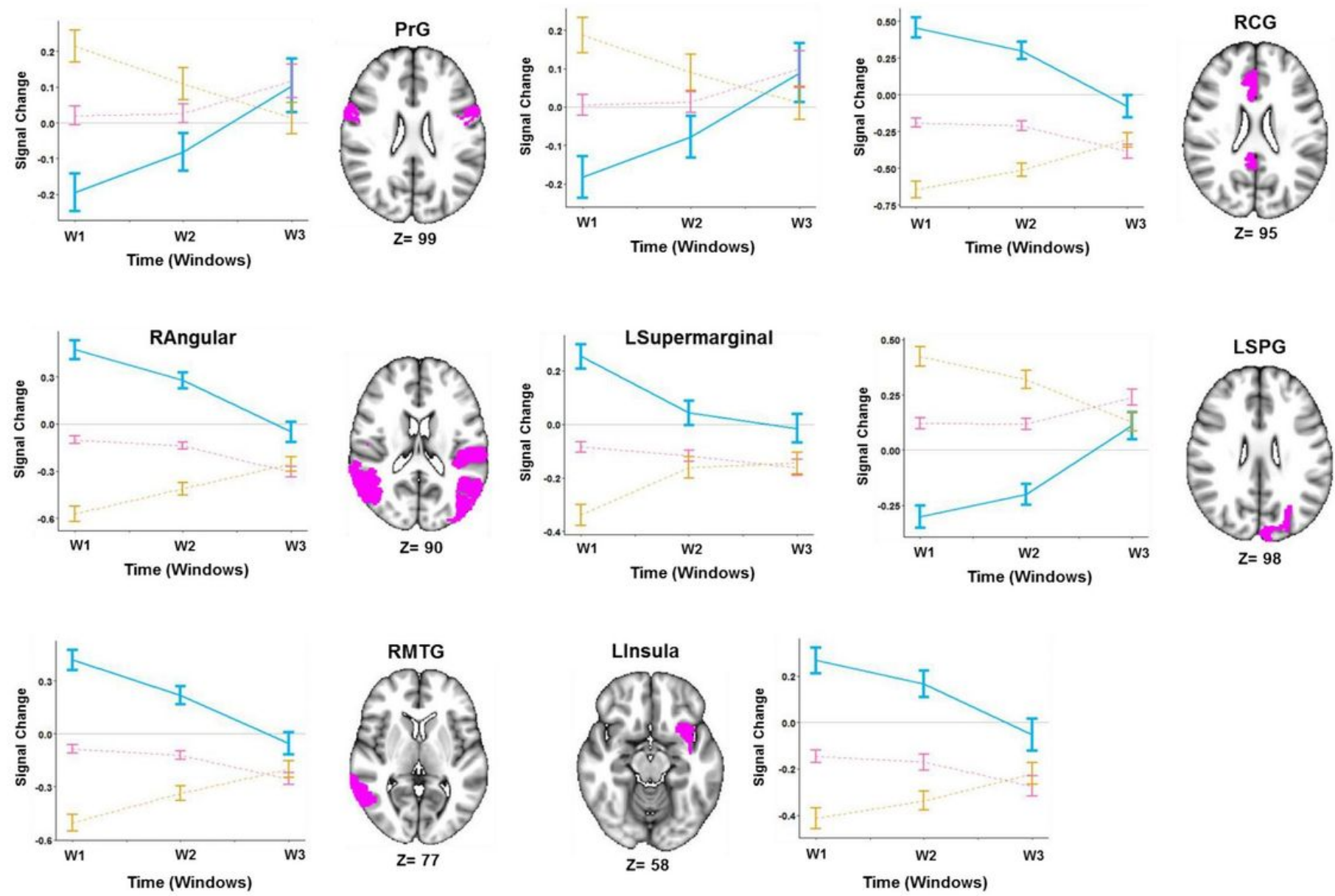

\section{Figure 5}

Temporal behavior of ROls with significant condition by time interactions in the response-inhibition linear mixed effects (LME) model. Lines show the average main effect of NoGo ((Drug Successful NoGo+Neutral Successful NoGo) or Go (Drug Successful Go+Neutral Successful Go)) inhibition condition in the Brainnetome (BNA) regions or the result of Go vs. NoGo contrast,, and error bars show the standard 
error of z-statistic values across 53 methamphetamine use disorders (MUD) at each temporal window (FDR corrected p-value<0.001). Abbreviation: MFG: middle frontal gyrus, RIFG (opercular): right opercular part of inferior frontal gyrus, PrG: precentral gyrus, RCG: right cingulate gyrus, LSPG: left superior parietal gyrus, RMTG: right middle temporal gyrus, RAngular: right angular gyrus, LInsula: left insula.

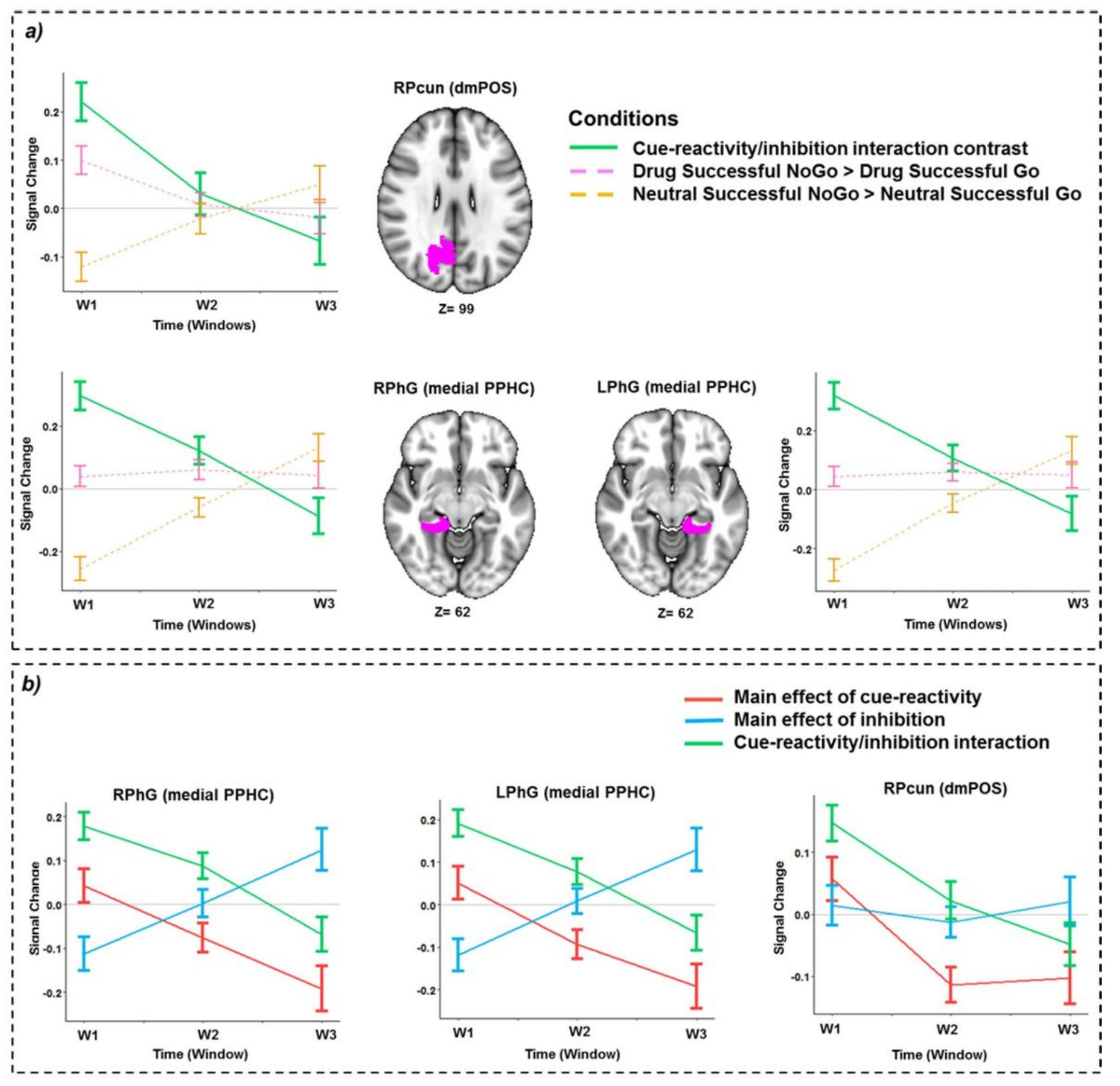

Figure 6

a) Temporal behavior of ROIs with significant condition by time interactions in the cuereactivity/response-inhibition interaction linear mixed effects (LME) model. Lines show the average main effect of Drug-related inhibition (Drug Successful NoGo-Drug Successful Go) or Neutral inhibition (Neutral 
Successful NoGo-Neutral Successful Go) interaction condition or the result of Cue-reactivity/response inhibition contrast in the Brainnetome (BNA) regions, and error bars show the standard error of z-statistic values across 53 methamphetamine use disorders (MUD) at each temporal window (FDR corrected $p$ value $<0.001)$. b) Temporal behavior of the main effects of cue-reactivity, response-inhibition and the interaction of cue-reactivity and response-inhibition contrasts in the ROls with significant condition by time interactions extracted from the cue-reactivity/response-inhibition interaction LME model (FDR corrected $p$-value $<0.001)$. The lines show mean parameter estimates, and the error bars show the standard error of z-statistic values across 53 methamphetamine use disorders (MUD). Abbreviation: RPcun (dmPOS): dorsomedial parietooccipital sulcus part of right precuneus, RPhG: right parahippocampal gyrus, LPhG: left parahippocampal gyrus.

\section{Supplementary Files}

This is a list of supplementary files associated with this preprint. Click to download.

- SupplementaryMaterials.docx 